\title{
Microwave type III bursts and pulsation groups
}

\author{
Z. Ning ${ }^{1,2}$, M. D. Ding ${ }^{1}$, H. A. Wu ${ }^{2}$, F. Y. Xu ${ }^{2}$, and X. Meng ${ }^{2}$ \\ 1 Department of Astronomy, Nanjing University, Nanjing 210093, PR China \\ e-mail: ningzongjun@hotmail.com \\ 2 Purple Mountain Observatory, Nanjing 210093, PR China
}

Received 3 September 2004 / Accepted 8 March 2005

\begin{abstract}
We analyse 22 continuous microwave reverse slope (RS) type III and 21 microwave pulsation groups observed by the Purple Mountain Observatory (PMO) spectrometer over the frequency range of 4.5-7.5 GHz from 1999-2003. The RS type III groups have an average of 12 bursts per group, a mean duration of $0.1-2 \mathrm{~s}$ around $6 \mathrm{GHz}$, a mean quasi-period of $2.0 \pm 0.6 \mathrm{~s}$ and a mean drift rate of $7.0 \mathrm{GHz} \mathrm{s}^{-1}$. The shortest quasi-period is measurable down to $0.05 \mathrm{~s}$ thanks to the high time resolution of this instrument. The microwave pulsation groups have an average of 22 events per group, a mean duration of 3-4 s and a mean quasi-period of $3.7 \pm 1.1 \mathrm{~s}$. The RS type III bursts have the same quasi-period as the upward type III bursts documented before. We discuss two possible origins of the quasi-periodicity of type III bursts. One is the quasi-periodic process in magnetic reconnection at the same site, and the other is spatially unresolved stochastic processes in solar flares.
\end{abstract}

Key words. Sun: radio radiation

\section{Introduction}

It is widely accepted that the energy released in solar activity comes from the magnetic field. Magnetic reconnection is thought to be the basic process of energy dissipation. More and more observations have shown evidence to support this viewpoint. Masuda et al. (1994) first observed a loop-top hard $\mathrm{X}$-ray source in a compact flare and interpreted it as evidence for magnetic reconnection above the loop top. Sui et al. (2003) found that the altitude distribution of temperature in both looptop and corona hard X-ray sources indicates the formation of a large-scale current sheet in the solar flare that occurred on 2002 April 15. Yokoyama \& Shibita (1995) observed X-ray jets and $\mathrm{H} \alpha$ surges that are thought to be produced by reconnection. Innes et al. (1997a) showed that the transition region explosive events are consistent with bi-directional jets produced by magnetic reconnection. They presented the time variation and spatial structure of the magnetic reconnection process for the first time. Recently, Ning et al. (2004) reported that transition region explosive events have a 3-5 min quasi-period, which suggests that the magnetic reconnection repeatedly takes place at the same site with a quasi-periodicity on the quiet Sun. Sui et al. (2003) found observational evidence of continuous reconnection at a flare loop top because both loop-top and corona hard $\mathrm{X}$-ray sources smoothly move upward with various speeds. Liu et al. (2004) showed that the footpoint and loop-top hard X-ray sources gradually move outside and upward respectively during a flare that occurred on 2003 November 3, which also indicates continuous reconnection in a flare. Therefore, it is an interesting question as to whether there is a quasi-periodicity in the continuous reconnection in solar active regions, and if there is, how long it lasts.

In the classical reconnection model for solar flares (e.g. Sturrock 1964), the released magnetic energy is quickly converted to thermal and kinetic energy of plasma and particles, which is reflected from the emission at various wavebands. Aschwanden et al. (1995) and Ning et al. (2000) showed that the solar type III burst pairs, both an upwardpropagating type III burst (with a negative frequency-time drift rate, $\mathrm{d} f / \mathrm{d} t<0$ ) and a downward-propagating reverse slope (RS) type III burst (with a positive frequency-time drift rate, $\mathrm{d} f / \mathrm{d} t>0$ ), are consistent with bi-directional electron beams produced by magnetic reconnection in flares. The quasiperiodicity of type III bursts could be helpful to understand the energy dissipation process, especially the quasi-periodicity of magnetic reconnection in the active region. Aschwanden et al. (1994) has statistically studied the quasi-periodicity of type III bursts in order to clarify the coherent-phase or randomphase acceleration of electron beams in solar flares. They found a total of 260 continuous type III groups observed by Ikarus (Zurich) in the frequency range of 100-500 MHz, during 359 flares in the years 1980-1983. On average, there are $13 \pm 9$ bursts per group, with a mean duration of $D=12 \pm 14 \mathrm{~s}$ per group, and a mean quasi-period of $P=2.0 \pm 1.2 \mathrm{~s}$. They mainly studied the upward type III bursts and their subclasses, type $\mathrm{J}$ and type $\mathrm{U}$ bursts in the metric wavelength. They concluded that the electron beams are accelerated upward by coherent-phase reconnection and with a quasi-period 
of $2.0 \mathrm{~s}$ during solar flares. It is an interesting question as to whether the downward electron beams, which generally produce microwave bursts (frequency $>1 \mathrm{GHz}$ ), especially RS type III bursts, have a quasi-period as well. The quasiperiod of RS type III bursts, which appear preferentially above $500 \mathrm{MHz}$ (Aschwanden et al. 1997), is still not well known. In this study, we attempt to determine the quasi-period of RS type III bursts around $6 \mathrm{GHz}$.

Mangeney \& Pick (1989) and Zhao et al. (1991) previously found that the quasi-periodicity ranges between 1 and $6 \mathrm{~s}$ in the type III bursts using Fourier transformation. They also concluded that this kind of quasi-periodicity occurs because of the quasi-periodic process in solar flares. Contrary to these investigations, Isliker (1996), who studied stochastic processes in flares, suggested that the interpretation of these empirical results not as simple as these authors suggest. It is possible that the quasi-periodic behaviour of type III bursts can be the signature of the stochastic process in solar flares. Isliker \& Benz (1994) did not find any hint of quasi-periodic behaviour for type III and narrow-band spike events. We will discuss these two kinds of interpretation for RS type III burst groups in this paper.

\section{Observations}

The data described here were obtained with the 4.5-7.5 GHz radio spectrometer of the Purple Mountain Observatory (PMO) in China. It has 300 frequency channels of $10 \mathrm{MHz}$ bandwidth each and a time resolution of $5 \mathrm{~ms}$. This spectrometer has obtained much valuable data during the last solar maximum cycle. It can detect more than 150 active events each year. Its daily working time is between 1:00-9:00 UT. Description of this instrument can be found in $\mathrm{Xu}$ et al. (2003). According to our observations, the main form of radio emission at this band is microwave type IV burst, which is the counterpart of decimetric (DCIM) bursts around $6 \mathrm{GHz}$. These type IV bursts are generally produced by solar flares that can also be detected in $\mathrm{H} \alpha$ or X-ray wavelengths. We observed fewer microwave type III bursts than type IV bursts.

We analyze a total of 22 groups of continuous microwave type III bursts and 21 groups of microwave pulsations observed by the PMO spectrometer during 40 solar flares in the years 1999-2003. Table 1 presents all the events studied in this paper. We classify the events into three types, RS-III, Blips and Pulsation, based on the $1-3 \mathrm{GHz}$ solar radio catalogue (Isliker \& Benz 1994). The corresponding X-Ray flares are presented if the bursts take place during the flare. All of the RS type III group or blips appear in the impulsive phase of X-ray flares, which is consistent with statistical studies in the metric wavelength (e.g. Kane 1974). According to the Solar Geophysical Data (SGD), one RS type III on 2002 April 14 and two pulsation groups on 1999 September 11 and December 16 occurred without any flares detected in $\mathrm{H} \alpha$ and X-ray. Possibly, the flares were too faint to be detected by present instruments. We also note that pulsations took place 20 min later in the B8.3 flare of 2003 June 21.

\subsection{Identification of events}

We identify the individual RS type III bursts based on the criterion used by Isliker \& Benz (1994) 1n the 1-3 GHz band. They showed that type III bursts have a duration of the order of $0.1-1 \mathrm{~s}$. The narrow band $(<100 \mathrm{MHz})$ type III bursts are called blips. We first identify the individual RS type III burst dependence in a mean duration of $0.1-2 \mathrm{~s}$ around $6 \mathrm{GHz}$, which is slightly longer than that at $1-3 \mathrm{GHz}$ (Isliker \& Benz 1994). Secondly, RS type III groups are identified by visual inspection of the broad-band dynamic spectra in the frequency range of $4.5-7.5 \mathrm{GHz}$ and by the measurement of the frequency-time drift rate. The frequency drift rate of each individual RS type III burst is calculated over its bandwidth, and the mean value of all RS type III bursts in Table 1 is about $7.0 \mathrm{GHz} \mathrm{s}^{-1}$ (in the range of $2.5-13 \mathrm{GHz} \mathrm{s}^{-1}$ ), which is much faster than metric type III bursts $\left(-100 \mathrm{kHz} \mathrm{s}^{-1}\right)$. Furthermore, we identified microwave pulsation events, which generally exhibit a more regular flux amplitude, a higher frequency drifting rate and a mean duration of 3-4 s around $6 \mathrm{GHz}$.

The RS type III and pulsation peaks often overlap a gradual component. Thus these peaks have a higher value than the background emission of the quiet sun. However, some peaks lie very near the noise level. As shown in Fig. 1, the burst peaks have to be larger than 3 times the standard deviation $(\sigma)$ of the noise, which is computed by the average profile in two windows on either side of the radio bursts, for example, between time period 05:15-05:25 and 05:45-05:55 (top panel in Fig. 1), $\sigma \sim 3$ (arbitrary units) for the event on April 142002. Then, we estimate the quasi-period $P$ in RS type III and pulsation groups from the formula as follows (Aschwanden et al. 1994)

$P=\frac{1}{N_{P}-1} \sum_{i=1}^{N_{P}-1} P_{i}$

where $N_{P}$ is the total number of RS type bursts in each group, $t_{i}$ is the peak time of each individual burst at a fixed frequency, $i=1,2, \ldots N_{P}$, and $P_{i}$ is the time interval between subsequent bursts, i.e., $P_{i}=t_{i}-t_{i-1}$. To quanlify the degree of periodicity in a time series, we calculate the standard deviation of the individual estimated quasi-period with the following formula for each event in Table 1

$\sigma_{P}=\sqrt{\frac{1}{N_{P}-2} \sum_{i=1}^{N_{P}-1}\left(P_{i}-P\right)^{2}}$.

The parameter $\sigma_{P} / P$, that is the standard deviation $\sigma_{P}$ normalized by the mean quasi-period $P$, is thought to be the "quality index" of these estimated quasi-periods. In other words, it can tell us, at least semi-quantitatively, how "strict" these quasi-periods are. For a strictly periodic behaviour, no scatter of the mean quasi-period is expected, i.e. $\sigma_{P}=0$ or $\sigma_{P} / P=0$. Therefore, the RS-III bursts on Aug. 251999 in Fig. 2 has a more strictly quasi-periodic behaviour than the Blips on Apr. 142002 in Fig. 1. The former (the quasi-period $P=2.0 \pm 0.6 \mathrm{~s}$ ) has a larger "quality index" of $\sigma_{P} / P=0.3$ than that of $\sigma_{P} / P=0.5$ for the latter Blips (the quasi-period $P=0.4 \pm 0.2 \mathrm{~s})$. 
Table 1. The data set used in this paper obtained at PMO (China) in 1999-2003.

\begin{tabular}{|c|c|c|c|c|c|c|c|c|}
\hline No. & Date & Type & Time (UT) & Number & Quasi-period & Mean drifting & Flare (start-max-end) & Flare type \\
\hline 1 & 25 Aug. 1999 & III & $0134-0135$ & 26 & $2.0 \pm 0.6 \mathrm{~s}$ & $3.5 \mathrm{GHz} \mathrm{s}^{-1}$ & $0132-0136-0140$ & M3.6 \\
\hline 2 & 11 Sep. 1999 & Puls. & $0259-0300$ & 9 & $1.2 \pm 0.4 \mathrm{~s}$ & & No flare patrol ${ }^{*}$ & \\
\hline 3 & 25 Oct. 1999 & III & $0629-0630$ & 8 & $4.4 \pm 1.4 \mathrm{~s}$ & $6.0 \mathrm{GHz} \mathrm{s}^{-1}$ & $0626-0631-0634$ & M1.7 \\
\hline 4 & 27 Oct. 1999 & III & $0428-0429$ & 3 & $1.0 \pm 0.3 \mathrm{~s}$ & $5.0 \mathrm{GHz} \mathrm{s}^{-1}$ & $0426-0430-0432$ & $\mathrm{C} 5.3$ \\
\hline 5 & 16 Dec. 1999 & Puls. & $0736-0737$ & 6 & $3.0 \pm 0.5 \mathrm{~s}$ & & No flare* & \\
\hline 6 & 23 Dec. 1999 & III & $0314-0315$ & 4 & $1.8 \pm 0.7 \mathrm{~s}$ & $5.5 \mathrm{GHz} \mathrm{s}^{-1}$ & $0312-0315-0318$ & C1.6 \\
\hline 7 & 27 Dec. 1999 & III & 0148-0149 & 4 & $4.1 \pm 1.3 \mathrm{~s}$ & $8.0 \mathrm{GHz} \mathrm{s}^{-1}$ & 0146-0154-0209 & M1.0 \\
\hline 8 & 20 Jan. 2000 & III & $0224-0225$ & 3 & $1.8 \pm 0.6 \mathrm{~s}$ & $11.0 \mathrm{GHz} \mathrm{s}^{-1}$ & $0225-0229-0230$ & $\mathrm{C}$ \\
\hline 9 & 06 Apr. 2000 & III & $0221-0222$ & 3 & $1.6 \pm 0.3 \mathrm{~s}$ & $3.2 \mathrm{GHz} \mathrm{s}^{-1}$ & $0218-0229-0238$ & M1.8 \\
\hline 10 & 08 Apr. 2000 & III & $0238-0239$ & 37 & $1.5 \pm 0.5 \mathrm{~s}$ & $15.0 \mathrm{GHz} \mathrm{s}^{-1}$ & $0234-0240-0250$ & M2.0 \\
\hline 11 & 27 Jul. 2000 & Puls. & $0407-0408$ & 4 & $2.0 \pm 0.8 \mathrm{~s}$ & & $0406-0410-0413$ & M2.4 \\
\hline 12 & 12 Nov. 2001 & Blips & $0755-0756$ & 10 & $2.8 \pm 0.6 \mathrm{~s}$ & $4.5 \mathrm{GHz} \mathrm{s}^{-1}$ & $0752-0757-0801$ & M1.6 \\
\hline 13 & 24 Nov. 2001 & III & $0456-0457$ & 3 & $4.4 \pm 0.9 \mathrm{~s}$ & $7.0 \mathrm{GHz} \mathrm{s}^{-1}$ & $0456-0501-0506$ & $\mathrm{C} 2.3$ \\
\hline 14 & 03 Dec. 2001 & Puls. & $0710-0711$ & 6 & $3.2 \pm 0.7 \mathrm{~s}$ & & $0706-0712-0716$ & C7.0 \\
\hline 15 & 11 Dec. 2001 & III & $0341-0342$ & 18 & $3.5 \pm 1.1 \mathrm{~s}$ & $3.7 \mathrm{GHz} \mathrm{s}^{-1}$ & $0320-0343-0347$ & $\mathrm{C} 3.4$ \\
\hline 16 & 19 Dec. 2001 & III & $0230-0231$ & 20 & $2.4 \pm 0.5 \mathrm{~s}$ & $4.2 \mathrm{GHz} \mathrm{s}^{-1}$ & $0229-0232-0236$ & $\mathrm{C} 4.2$ \\
\hline 17 & 13 Jan. 2002 & Puls. & $0536-0537$ & 25 & $1.4 \pm 0.3 \mathrm{~s}$ & & $0534-0538-0542$ & $\mathrm{C} 3.5$ \\
\hline 18 & 14 Apr. 2002 & Blips & $0536-0537$ & 36 & $0.4 \pm 0.2 \mathrm{~s}$ & $3.0 \mathrm{GHz} \mathrm{s}^{-1}$ & No flare* & \\
\hline 19 & 21 Apr. 2002 & Puls. & $0129-0130$ & 29 & $4.4 \pm 1.3 \mathrm{~s}$ & & $0043-0151-0238$ & $\mathrm{X} 1.5$ \\
\hline 20 & 21 Apr. 2002 & Puls. & $0152-0153$ & 19 & $6.0 \pm 2.1 \mathrm{~s}$ & & $0043-0151-0238$ & $\mathrm{X} 1.5$ \\
\hline 21 & 21 Apr. 2002 & Puls. & $0226-0227$ & 9 & $6.0 \pm 2.8 \mathrm{~s}$ & & $0043-0151-0238$ & $\mathrm{X} 1.5$ \\
\hline 22 & 21 Apr. 2002 & Puls. & $0228-0229$ & 19 & $3.8 \pm 1.4 \mathrm{~s}$ & & $0043-0151-0238$ & $\mathrm{X} 1.5$ \\
\hline 23 & 01 Jun. 2002 & Puls. & $0353-0354$ & 19 & $3.0 \pm 0.6 \mathrm{~s}$ & & $0350-0357-0401$ & M1.5 \\
\hline 24 & 17 Jul. 2002 & Puls. & $0712-0713$ & 12 & $2.3 \pm 1.3 \mathrm{~s}$ & & $0658-0713-0719$ & M8.5 \\
\hline 25 & 11 Sep. 2002 & III & $0730-0731$ & 4 & $1.4 \pm 0.6 \mathrm{~s}$ & $7.0 \mathrm{GHz} \mathrm{s}^{-1}$ & $0726-0735-0742$ & M2.2 \\
\hline 26 & 11 Sep. 2002 & III & $0731-0732$ & 4 & $1.4 \pm 0.8 \mathrm{~s}$ & $6.2 \mathrm{GHz} \mathrm{s}^{-1}$ & $0726-0735-0742$ & M2.2 \\
\hline 27 & 29 Sep. 2002 & Puls. & $0636-0637$ & 5 & $4.4 \pm 1.4 \mathrm{~s}$ & & $0632-0639-0641$ & M2.6 \\
\hline 28 & 16 Jan. 2003 & III & 0104-0107 & 36 & $2.3 \pm 0.6 \mathrm{~s}$ & $8.0 \mathrm{GHz} \mathrm{s}^{-1}$ & $0104-0108-0115$ & C5.3 \\
\hline 29 & 21 Jan. 2003 & III & $0225-0226$ & 3 & $1.8 \pm 0.5 \mathrm{~s}$ & $2.5 \mathrm{GHz} \mathrm{s}^{-1}$ & $0223-0228-0233$ & $\mathrm{C} 8.1$ \\
\hline 30 & 06 Feb. 2003 & Puls. & $0332-0333$ & 3 & $3.0 \pm 0.9 \mathrm{~s}$ & & $0330-0349-0401$ & M1.2 \\
\hline 31 & 06 Feb. 2003 & Puls. & $0346-0347$ & 14 & $4.0 \pm 1.5 \mathrm{~s}$ & & $0330-0349-0401$ & M1.2 \\
\hline 32 & 06 Feb. 2003 & Puls. & $0348-0349$ & 12 & $3.5 \pm 2.1 \mathrm{~s}$ & & $0330-0349-0401$ & M1.2 \\
\hline 33 & 06 Feb. 2003 & Puls. & $0350-0351$ & 12 & $5.0 \pm 2.2 \mathrm{~s}$ & & $0330-0349-0401$ & M1.2 \\
\hline 34 & 18 Mar. 2003 & Blips & $0559-0600$ & 4 & $0.05 \pm 0.02 \mathrm{~s}$ & $10 \mathrm{GHz} \mathrm{s}^{-1}$ & $0551-0600-0602$ & M2.5 \\
\hline 35 & 27 May 2003 & Puls. & $0253-0254$ & 11 & $4.0 \pm 1.7 \mathrm{~s}$ & & $0240-0306-0321$ & M1.4 \\
\hline 36 & 31 May 2003 & III & $0218-0219$ & 9 & $2.1 \pm 0.8 \mathrm{~s}$ & 13.0 $\mathrm{GHz} \mathrm{s}^{-1}$ & $0213-0224-0240$ & M9.3 \\
\hline 37 & 31 May 2003 & III & $0218-0219$ & 6 & $2.1 \pm 0.7 \mathrm{~s}$ & $13.0 \mathrm{GHz} \mathrm{s}^{-1}$ & $0213-0224-0240$ & M9.3 \\
\hline 38 & 21 Jun. 2003 & Puls. & $0338-0339$ & 7 & $4.0 \pm 1.6 \mathrm{~s}$ & & $0303-0309-0316$ & B8.3 \\
\hline 39 & 22 Oct. 2003 & Puls. & $0328-0329$ & 13 & $5.0 \pm 2.9 \mathrm{~s}$ & & $0328-0351-0352$ & M3.7 \\
\hline 40 & 23 Oct. 2003 & III & $0238-0239$ & 19 & $2.5 \pm 0.6 \mathrm{~s}$ & $3.0 \mathrm{GHz} \mathrm{s}^{-1}$ & $0235-0241-0244$ & M2.0 \\
\hline 41 & 25 Oct. 2003 & Puls. & $0547-0548$ & 11 & $3.5 \pm 0.9 \mathrm{~s}$ & & $0544-0553-0626$ & M1.7 \\
\hline 42 & 26 Oct. 2003 & Puls. & 0508-0509 & 9 & $2.3 \pm 0.7 \mathrm{~s}$ & & $0557-0654-0733$ & $\mathrm{X} 1.2$ \\
\hline 43 & 19 Nov. 2003 & III & 0400-0401 & 5 & $2.6 \pm 0.5 \mathrm{~s}$ & $8.0 \mathrm{GHz} \mathrm{s}^{-1}$ & $0355-0401-0406$ & M1.7 \\
\hline
\end{tabular}

* No flare records on Solar-Geophysical data.

\subsection{RS type III groups}

Figure 1 shows one sample of these RS type III groups on 2002 April 14. It has in total more than 36 individual RS blips with a mean drift rate of $3.0 \mathrm{GHz} \mathrm{s}^{-1}$. They have a short quasi-period of $0.4 \mathrm{~s}$. Figure 2 shows an RS type III group preceding a microwave type V burst on 1999 August 25, which is a unique example in our observations during 1999-2003. This group has 26 individual RS type III bursts with a mean drift rate of $3.5 \mathrm{GHz} \mathrm{s}^{-1}$. The bursts show a quasi-period of $2.0 \mathrm{~s}$. The dynamic spectra show that these RS type III bursts are fainter at higher frequency, almostly disappearing 

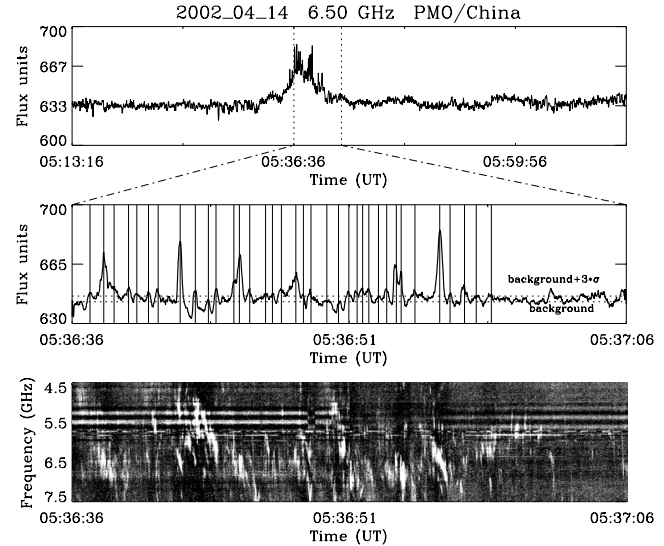

Fig. 1. An example of RS blips (narrow-band type III bursts) on 2002 April 14, around 0536 UT observed by PMO, without any flare detected by $\mathrm{H} \alpha$ and X-ray (from SGD). Top panel: time profile at a fixed frequency of $5.5 \mathrm{GHz}$. The flux is given in arbitrary units. Middle panel: $36 \mathrm{RS}$ blips identified by vertical lines on the time profile at a fixed frequency of $5.5 \mathrm{GHz}$. The mean quasi-period of bursts is about $0.4 \pm 0.2 \mathrm{~s}$. $\sigma$ gives the standard deviation of the noise level before or after solar radio bursts. Bottom panel: dynamic spectra of RS blips groups over the of $4.5-7.5 \mathrm{GHz}$ frequency band.
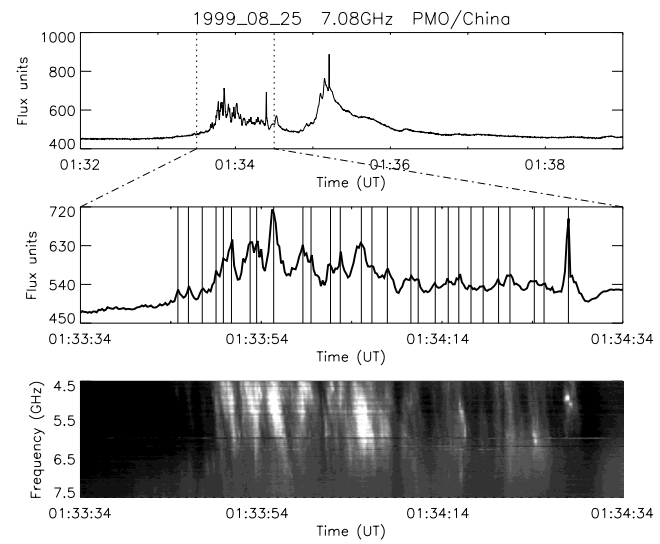

Fig. 2. A unique example has RS type III groups preceding microwave type V continuum for the flare of 1999 August 25. 26 individual RS type III bursts are identified by solid lines with a mean quasi-period of $2.0 \pm 0.6 \mathrm{~s}$. The illustration is similar to Fig. 1 .

around $7 \mathrm{GHz}$, while the following type $\mathrm{V}$ continuum does not. Solar type V bursts are dynamic continua generally following type III bursts (e.g. Wild 1959). Type V continua are thought to be produced by the same electrons trapped in loops as those moving along magnetic field lines to generate the type III bursts discussed earlier. According to SGD, metric type $\mathrm{V}$ bursts are detected by LEAR and PALE in the frequency range of $30-80 \mathrm{MHz}$ or $25-75 \mathrm{MHz}$ at the same quasi-period of 0133-0140 UT.

Figure 3 shows a sample of RS type III groups in the impulsive phase of microwave type IV bursts for an M2.2 flare on 2002 September 11. There are two RS type III groups each of four bursts. They have a quasi-period of $1.4 \mathrm{~s}$ and a mean drift rate of $7.0 \mathrm{GHz} \mathrm{s}^{-1}$. Figure 4 shows an example of an RS type III group in the decay phase of microwave type IV bursts for an M2.0 flare on 2000 April 8. Note that
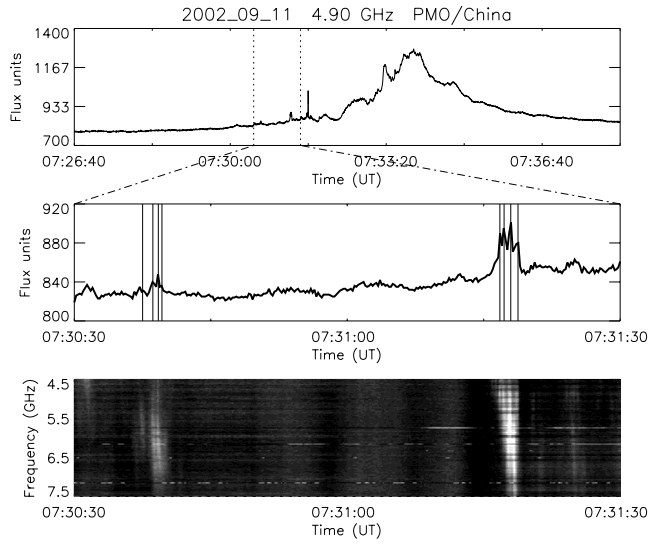

Fig. 3. An example of two RS type III groups in the impulsive phase of microwave type IV bursts for the flare of 2002 September 11. Two groups of four individual RS type III bursts each are identified by solid lines with a mean quasi-period of $1.4 \pm 0.6 \mathrm{~s}$. This illustration is similar to Fig. 1.

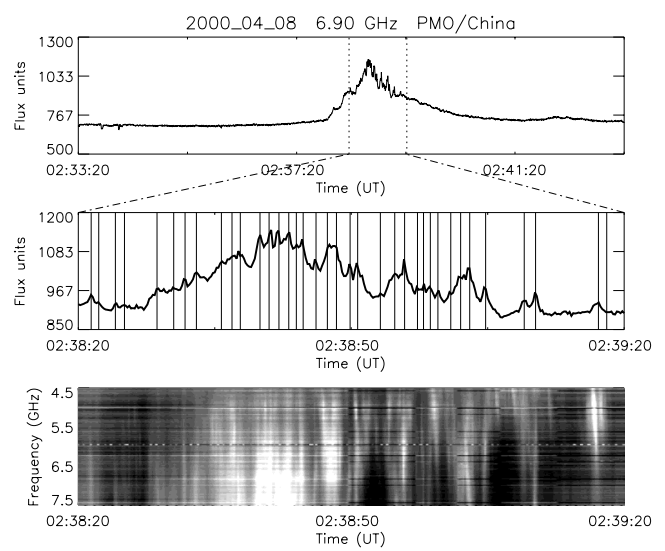

Fig. 4. An example of RS type III group in the decay phase of microwave type IV bursts for the flare of 2000 April 8. 37 individual RS type III bursts are identified by solid lines with a mean quasi-period of $1.5 \pm 0.5 \mathrm{~s}$. The illustration is similar to Fig. 1 .

the bursts are still in the impulsive phase of the X-ray flare. There are in total 37 individual RS type III bursts with a quasiperiod of $1.5 \mathrm{~s}$ and a mean drift rate of $15.0 \mathrm{GHz} \mathrm{s}^{-1}$. Figure 5 shows an RS type III group without any other microwave radio emission for a C5.3 flare on 1999 October 27. There are 5 RS type III bursts with a quasi-period of $1.0 \mathrm{~s}$ and a mean drift rate of $5.0 \mathrm{GHz} \mathrm{s}^{-1}$.

\subsection{Microwave pulsation groups}

The pulsation bursts have dynamic spectra almost without any frequency drift. This is because either the pulsation emission is simultaneously generated on a wide bandwidth or that the pulsation is a special sub-class of type III bursts with a very rapidly drifting rate which cannot be detected by the present instruments. We identify the pulsation groups as well as microwave type III burst groups. Isliker \& Benz (1994) showed that the pulsation bursts have a shorter duration (less than $0.1 \mathrm{~s}$ ) than type III bursts over the $1-3 \mathrm{GHz}$ band. In our data, we identify a individual pulsation with a mean duration of 3-4 s, 

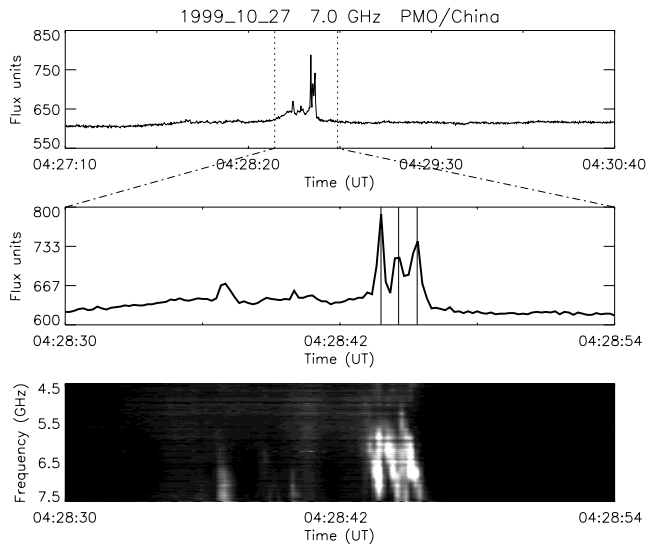

Fig. 5. An example of an RS type III group without any other microwave emission for the flare of 1999 October 27. 5 individual RS type III bursts take place and the last 3 identified by solid lines have a mean quasi-period of $1.0 \pm 0.3 \mathrm{~s}$. The illustration is similar to Fig. 1.
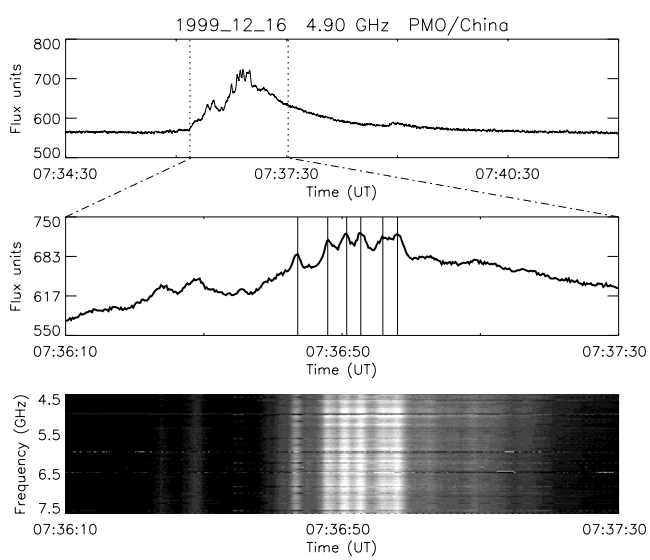

Fig. 6. An example of microwave pulsation group in the impulsive phase of microwave type IV bursts for the flare of 1999 December 16. 8 individual pulses occur and the last 6 identified by solid lines have a mean quasi-period of $3.0 \pm 0.5 \mathrm{~s}$. The illustration is similar to Fig. 1 .

which is longer than RS type III bursts around $6 \mathrm{GHz}$. In Table 1, we present 21 groups of microwave pulsations. They have a mean quasi-period of $3.7 \mathrm{~s}$ (in the ranges of 1.2-6.0 s). Figure 6 shows an example of a pulsation group in the impulsive phase of microwave type IV bursts without any detected flare on 1999 December 16 (from SGD). It has in total 8 pulsations, the latter 6 having a quasi-period of $3.0 \mathrm{~s}$. Figure 7 presents another pulsation group in the decay phase of microwave type IV bursts for an M1.5 flare on 2002 June 1. It has a 19 individual pulsations with a quasi-period of $3.0 \mathrm{~s}$.

Although the physical reason for pulsations is still controversial, it is interesting that these pulsation groups show a quasi-period of $3.7 \mathrm{~s}$, which is similar to the behaviour of RS type III groups around $6 \mathrm{GHz}$.

\subsection{A special microwave type III group}

Figure 8 shows a special microwave type III group. Although the individual burst shows a very short life time (less than $0.05 \mathrm{~s}$ ) at a fixed frequency, we still regard the group as
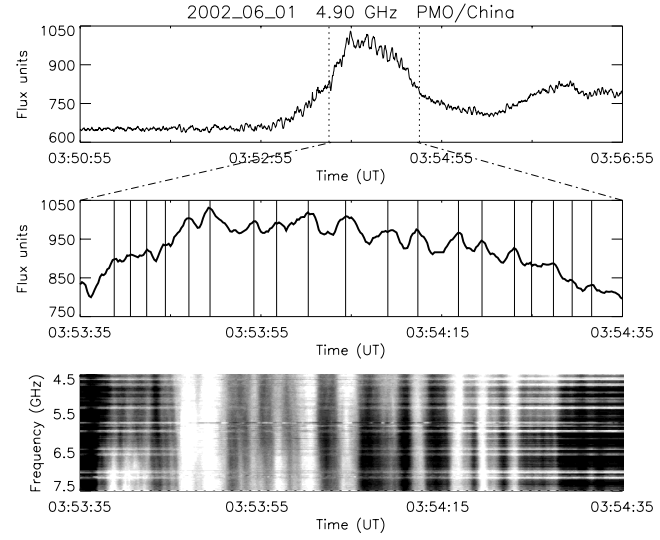

Fig. 7. An example of a microwave pulsation group in the decay phase of microwave type IV bursts for the flare of 2002 June 1.19 individual pulses are identified by solid lines with a mean quasi-period of $3.0 \pm$ $0.6 \mathrm{~s}$. The illustration is similar to Fig. 1.

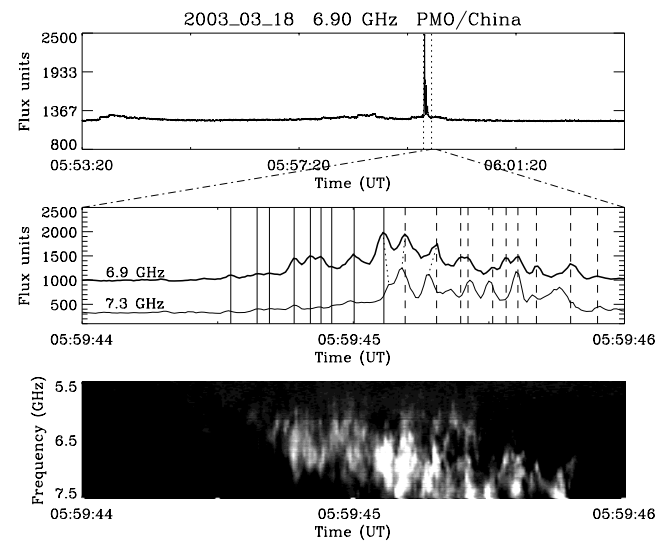

Fig. 8. A special microwave type III group mixed RS type III burst (solid lines) preceding the upward type III bursts (dashed lines) for the flare of 2002 March 18. Both RS and upward type III bursts have a very short quasi-period of $0.05 \mathrm{~s}$. The $7.3 \mathrm{Ghz}$ profile is overplotted after subtracting 700 flux units. One RS type III and two upward type III bursts are shown with dotted lines. The illustration is similar to Fig. 1.

type III bursts due to their frequency drift. There are more than 9 RS type III bursts (solid lines) preceding the upward type III bursts (dashed lines) during the flare on 2002 March 18. In order to determine the frequency drifting, the event is plotted in two different frequencies. Three drifting structures are plotted with dotted lines. RS type III bursts, as well as the following upward type III bursts, have a short mean quasi-period of up to $0.05 \mathrm{~s}$. It is interesting that upward type III bursts follow the RS type III bursts in time. They could be the fine structures around $7 \mathrm{GHz}$, which correspond to the fast evolution process of plasma in the low corona. It is possible that the following upward type III bursts might be produced by the same electron beams that first generate RS type III bursts before the mirror effect. From our observations, it is difficult to support or rule out this possibility. 


\section{Results and discussion}

We have presented the quasi-periodicity of RS type III bursts and pulsations around $6 \mathrm{GHz}$ in this paper. We found 22 continuous RS type III groups with an average of 12 bursts per group, a mean duration of $0.1-2 \mathrm{~s}$ per bursts, a mean drifting rate of $7.0 \mathrm{GHz} \mathrm{s}^{-1}$ and a mean quasi-period of $2.0 \pm 0.6 \mathrm{~s}$. As shown in Table 1, the longest quasi-period is $4.4 \mathrm{~s}$ and the shortest is $0.05 \mathrm{~s}$. The 21 continuous microwave pulsation groups have an average of 12 events per group, but a mean duration of 3-4 s per bursts, and a mean quasi-period of $3.7 \pm 1.1 \mathrm{~s}$. Here, the standard deviation is the average value of the standard deviation for the individual estimated quasi-period, which is computed for each event in Table 1 with the formula noted earlier.

The issue of whether the energy release in a solar flare is triggered at a critical place and spreads randomly, or whether the energy disspation is controlled by a global condition with a quasi-period, is still controversial. As mentioned earlier, Mangeney \& Pick (1989) and Zhao et al. (1991) searched for quasi-periodic behaviours in type III events by estimating the power-spectrum (using Fourier transforms), and they found that the typical quasi-periodicities are between 1 and $6 \mathrm{~s}$. Later, Aschwanden et al. (1994) made a statistical study of a total of 260 continuous type III groups. They found upward type III bursts with a $2.0 \mathrm{~s}$ quasi-period. These authors concluded that the electron beams are accelerated upward through the corona by the coherent-phase instead of random-phase during flares. The present observations of RS type III bursts with a $2.0 \mathrm{~s}$ quasi-period indicate that the electron beams are accelerated downward with a quasi-period as well. This is reminiscent of magnetic reconnection to accelerate the electron beams upward and downward simultaneously with a quasi-period of $2.0 \mathrm{~s}$ in active regions.

On the other hand, there is also an argument that the solar flare processes could be random, without a quasi-period (e.g. Isliker 1996). What has been found in the observations and termed as "periodic" or "almost periodic" can as well be the signature of temporal correlations in a stochastic flare model. At present, it is very hard to establish whether the solar flare processes are random or quasi-periodic from the available observations.

\subsection{Are solar flares or magnetic reconnections quasi-periodic processes?}

\subsubsection{Quasi-periodicity of magnetic reconnection in active regions}

In active regions, solar flares represent an explosive conversion of stored magnetic energy by reconnection into other forms. Sui et al. (2003) and Liu et al. (2004) found evidence of continuous reconnection in solar flares because of continuous outward motion of hard X-ray footpoint sources and upward motion of the loop top source. As mentioned earlier, both upward and RS continuous type III bursts have a quasi-period of $2.0 \mathrm{~s}$ in each group, which is also evidence that continuous reconnection accelerates the electron beams upward and downward by coherent-phase reconnection quasi-periodically. Although our radio observations provide no source images on the solar surface, both upward and RS type III bursts show similar dynamic spectra in each group. This phenomenon implies the possibility that continuous magnetic reconnection repeatedly takes place at the same site with a quasi-period of $2.0 \mathrm{~s}$ during solar flares. The dynamic spectra of type III bursts could be random instead of quasi-period when the electron beams are from various sites in active regions.

Nakajima et al. (1983) reported that the significant systematic differences between the times of peak intensities of microwave, hard X-ray and $\gamma$-ray line emissions suggest that two kinds of acceleration take place successively and repeatedly within a few seconds. They found that the bursts were composed of seven successive pulses on 1980 June 7 and four successive pulses on 1980 June 21 , both with a quasi-periodicity of $\sim 8 \mathrm{~s}$. The authors suggest that two kinds of acceleration take place repeatedly during the impulsive bursts, in order to interpret the quasi-periodicity of pulses and systematic differences between the peak times of microwave, hard X-ray and $\gamma$-ray line emissions. Both electrons and ions are preferentially accelerated with a quasi-periodic of $\sim 8 \mathrm{~s}$ which is the same order as the time interval between successive hard X-Ray pulses.

\subsubsection{Quasi-periodicity of magnetic reconnection in quiet regions}

Transition region explosive events are one of the best-studied dynamic phenomena in quiet regions of the Sun (e.g. Brueckner \& Bartoe 1983; Dere et al. 1989; Dere 1994; Chae et al. 1998). They are detected most frequently in transition region lines formed typically around $10^{5} \mathrm{~K}$, and rarely in lines formed below $3 \times 10^{4} \mathrm{~K}$ or above $3 \times 10^{5} \mathrm{~K}$ (e.g. Innes et al. 1997b). Explosive events are characterized by broad line profiles with high velocity components of typically $\sim 100 \mathrm{~km} \mathrm{~s}^{-1}$, a special size of $\sim 2 \operatorname{arcsec}(\sim 1500 \mathrm{~km})$, and an average lifetime of $\sim 1 \mathrm{~min}$. Since they were discovered, explosive events were considered to be a manifestation of magnetic reconnection occurring in the quiet Sun (e.g. Porter \& Dere 1991; Dere et al. 1991; Dere 1994). Base on the joint observations with the Solar Ultraviolet Measurement of Emitted Radiation (SUMER) spectrograph on board the Solar and Heliospheric Observatory (SOHO) and the Big Bear Solar Observatory (BBSO), Chae et al. (1998) found that explosive events tend to occur above regions with weak and mixed polarity magnetic flux on the photosphere, which further prove that explosive events represent magnetic reconnection in the transition region of the quiet Sun. From the observations of SUMER, Innes et al. (1997a) showed that the time variation and spatial structure of explosive events are consistent with the bi-directional plasma jets produced by magnetic reconnection. Quite recently, Ning et al. (2004) reported that explosive events repeatedly take place at the same site with a 3-5 min quasi-period, which suggests that magnetic reconnection has a quasi-period of 3-5 min in the transition region of the quiet Sun. 


\subsubsection{Memory property of magnetic reconnection at the same site}

The observations show that magnetic reconnection takes place repeatedly with a quasi-periodicity at the same site in both quiet and active regions. This property suggests that magnetic reconnection has a memory of its site. The following magnetic reconnection prefers to occur at the same site as the previous one. However, it is interesting to ask why the quasi-period is different in the transition region of the quiet Sun to that in the corona of the active region. This implies that the magnetic reconnection quasi-periodicity might be modulated by its surrounding environments. There are two types of oscillations (e.g. Lites et al. 1993) in the transition region of the quiet Sun, which are generally thought to be due to propagation of waves with a quasi-period of 3- or 5-min (e.g. Lou 1995; Banerjee et al. 2001). These waves might be able to modulate the quasi-period of magnetic reconnection there. Sakai \& Washimi (1982) and Sakai et al. (1984) proposed a theoretical model of forced reconnection caused by fast magnetosonic waves. However, it is unclear what effect modulates continuous reconnection with a quasi-period of $2 \mathrm{~s}$ or $8 \mathrm{~s}$ in solar flares. The quasi-periodicity of continuous reconnection needs to be checked further by observations with spatial resolution in the future.

\subsection{Are solar flares random processes?}

A quasi-periodicity in both upward and RS type III groups implies a quasi-periodicity in the acceleration mechanism if all these type III bursts originate from the same site. We cannot simply conclude that these electron beams for quasi-periodic type III bursts originate from the same site on the Sun, because the present observations provide poor spatial resolution. On the other hand, this interpretation relies on the model of a large scale current sheet, which is generally thought to be highly unstable and to be immediately fragmented into small current sheets. Emission from solar flares is fragmented in space and time, which favors the picture of multiple, small-scale (spatially unresolved) reconnections, called elementary bursts (e.g. LaRosa \& Moore 1993; Ding et al. 2001). It is possible that the quasi-periodicity of type III events is an observational artifact due to the overlapping of a large number of elementary bursts from different sites.

Isliker (1996) has studied the stochastic flare model in detail. He reported that the stochastic models for solar flares are compatible with the past and present observational results with high temporal resolution. Isliker thought that the many isolated peaks in the power spectra form differentiated time series of type III events, which Mangeney \& Pick (1989) considered to be indicative of quasi-periodic process, and suggested that they can be explained as the signature of a stochastic process. The empirical results of Aschwanden et al. (1994) can also be reproduced by the stochastic flare model. Isliker \& Benz (1994) find no hint for quasi-periodic behaviours and for finite dimensions in the power-spectrum and correlation-dimension estimates for type III and narrow-band spikes events. Therefore, it is also possible that the present observations of RS type III groups are signatures of the stochastic processes around $6 \mathrm{GHz}$. In order to discriminate between the two possible scenarios, we need to perform observations with a much longer time interval and an improved time resolution.

Acknowledgements. We would like to thank the referee for many valuable comments, which led to an improvement of the paper. This work was also supported by NKBRSF under grant G20000784, by NSFC under grant 10025315, 10221001, 10333030, 10279025 and 10333040, a grant form TRAPOYT, a JIANG-SUN-SHENG (2004) postdoctoral fund 0201003401 and a postdoctoral grant 0201003005 .

\section{References}

Aschwanden, M. J., Benz, A. O., \& Montello, M. L. 1994, ApJ, 431, 432

Aschwanden, M. J., Benz, A. O., Dennis, B. R., \& Schwartz, R. A. 1995, ApJ, 455, 347

Aschwanden, M. J., \& Benz, A. O. 1997, ApJ, 480, 825

Banerjee, D., O'Shea, E., Doyle, J. G., \& Goossens, M. 2001, A\&A, 371,1137

Brueckner, G. E., \& Bartoe, J.-D. F. 1983, ApJ, 272, 329

Chae, J., Wang, H., Lee, C., Goode, P. R., \& Schuehle, U. 1998, ApJ, 497, L109

Dere, K. P. 1994, Adv. Space Res., 14, 13

Dere, K. P., Bartoe, J.-D. F., \& Brueckner, G. E. 1989, Sol. Phys., 123, 41

Dere, K. P., Bartoe, J.-D. F., Brueckner, G. E., Ewing, J., \& Lund, P. 1991, J. Geophys. Res., 96, 9399

Ding, M. D., Qiu, J., Wang, H., \& Goode, P. R. 2001, ApJ, 552, 340

Larosa, T. N., \& Moore, R. L. 1993, ApJ, 418, 912

Lou, Y. 1995, MNRAS, 274, L1

Lites, B. W., Rutten, R. J., \& Kalkofen, W. 1993, ApJ, 414, 345

Liu, W., Jiang, Y. W., Liu, S., \& Petrosian, V. 2004, ApJ, submisstion

Innes, D. E., Inhester, B., Axford, W. I., \& Willhelm, K. 1997a, Nature, 386, 811

Innes, D. E., Brekke, P., Germerott, D., \& Wilhelm, K. 1997b, Sol. Phys., 175, 341

Isliker, H. 1996, A\&A, 310, 672

Isliker, H., \& Benz, A. O. 1994, A\&AS, 104, 145

Kane, S. R. 1974, Coronal Disturbances, 105

Masuda, S., Kosugi, T., Hara, H., Tsuneta, S., \& Ogawara, Y. 1994, Nature, 371, 495

Mangeney, A., \& Pick, M. 1989, A\&A, 224, 242

Nakajima, H., Kosugi, T., Kai, K., \& Enome, S. 1983, Nature, 305, 292

Ning, Z., Fu, Q., \& Lu, Q. 2000, Sol. Phys., 194, 137

Ning, Z., Innes, D. E., \& Solanki, S. K. 2004, A\&A, 419, 1141

Porter, J. G., \& Dere, K. P. 1991, ApJ, 370, 775

Sakai, J., Tajima, T., \& Brunel, F. 1984, Sol. Phys., 91, 103

Sakai, J.-I., \& Washimi, H. 1982, ApJ, 258, 823

Sturrock, P. A., \& Coppi, B. 1966, ApJ, 143, 3

Sui, L., \& Holman, G. D. 2003, ApJ, 596, L251

Xu, F., Xu, Z., Huang, G., et al. 2003, Sol. Phys., 216, 273

Yokoyama, T., \& Shibata, K. 1995, Nature, 375, 42

Wild, J. P., Sheridan, K. V., \& Trent, G. H. 1959, URSI Symp., 1, Paris Symposium on Radio Astronomy, IAU Symp., 9, 176

Zhao, R.-Y., Mangeney, A., \& Pick, M. 1991, A\&A, 241, 183 